\title{
Solutions of Electromagnetics Problems Involving Hundreds of Millions of Unknowns with Parallel Multilevel Fast Multipole Algorithm ${ }^{\dagger}$
}

\author{
Özgür Ergül ${ }^{1,2}$ and Levent Gürel ${ }^{1,2 *}$ \\ ${ }^{1}$ Department of Electrical and Electronics Engineering \\ ${ }^{2}$ Computational Electromagnetics Research Center (BiLCEM) \\ Bilkent University, TR-06800, Bilkent, Ankara, Turkey \\ \{ergul,lgurel\}@ee.bilkent.edu.tr
}

\section{Introduction}

We present the solution of extremely large electromagnetics problems formulated with surface integral equations (SIEs) and discretized with hundreds of millions of unknowns. Scattering and radiation problems involving three-dimensional closed metallic objects are formulated rigorously by using the combined-field integral equation (CFIE). Surfaces are discretized with small triangles, on which the Rao-Wilton-Glisson (RWG) functions are defined to expand the induced electric current and to test the boundary conditions for the tangential electric and magnetic fields. Discretizations of large objects with dimensions of hundreds of wavelengths lead to dense matrix equations with hundreds of millions of unknowns. Solutions are performed iteratively, where the matrix-vector multiplications are performed efficiently by using the multilevel fast multipole algorithm (MLFMA) [1]. Solutions are also parallelized on a cluster of computers using a hierarchical partitioning strategy [2], which is well suited for the multilevel structure of MLFMA. Accuracy and efficiency of the implementation are demonstrated on electromagnetic problems involving as many as $\mathbf{2 0 5}$ million unknowns, which are the largest integral-equation problems ever solved in the literature.

\section{Multilevel Fast Multipole Algorithm}

MLFMA reduces the complexity of the matrix-vector multiplications required by iterative solvers from $\mathcal{O}\left(N^{2}\right)$ to $\mathcal{O}(N \log N)$. A tree structure is constructed by placing the object in a cubic box and recursively dividing the computational domain into subboxes (clusters). Interactions between the clusters are calculated by translating the radiated fields of the clusters into incoming fields to other clusters. Without losing generality, consider a smooth object with an electrical dimension of $k D$, where $k=2 \pi / \lambda$ is the wavenumber. Discretization (triangulation) of the object with $\lambda / 10$ mesh size leads to $N$ unknowns, where $N=\mathcal{O}\left(k^{2} D^{2}\right)$. A multilevel tree structure with $L=\mathcal{O}(\log N)$ levels is obtained by considering nonempty boxes, i.e., clusters. At level $l$ from 1 to $L$, the number of clusters can be approximated as $N_{l} \approx 4^{(1-l)} N_{1}$, where $N_{1}=\mathcal{O}(N)$. In order the calculate the interactions between the clusters, radiated and incoming fields are defined and sampled on the unit sphere. The sampling rate depends on the cluster size as measured by the wavelength, and the total number of samples per cluster can be approximated as $S_{l} \approx 4^{(l-1)} S_{1}$, where $S_{1}=\mathcal{O}(1)$. We note that the complexity at level $l$ is proportional to the product of the number of clusters and the number of samples, i.e., $N_{l} S_{l} \approx N_{1} S_{1}=\mathcal{O}(N)$. Hence, all levels of MLFMA have equal importance with $\mathcal{O}(N)$ complexity in terms of processing time and memory.

\footnotetext{
${ }^{\dagger}$ This work was supported by the Scientific and Technical Research Council of Turkey (TUBITAK) under Research Grants 105E172 and 107E136, by the Turkish Academy of Sciences in the framework of the Young Scientist Award Program (LG/TUBA-GEBIP/2002-1-12), and by contracts from ASELSAN and SSM.
} 


\section{Hierarchical Parallelization of MLFMA}

For the solution of very large problems, MLFMA running on a single processor may not be sufficient, and parallelization is required. Unfortunately, MLFMA involves a complicated tree structure, which is difficult to distribute among multiple processors. Lower levels of the tree structure involve large numbers of clusters with coarsely-sampled radiated and incoming fields, while the higher levels involve small numbers of clusters with fine samplings. Recently, we developed a hierarchical partitioning strategy, which is based on distributing both clusters and field samples at each level by considering the numbers of clusters and samples [2]. This strategy improves the parallelization efficiency, compared to previous parallelization approaches that are based on distributing either clusters or field samples at a level.

Consider the parallelization of MLFMA on a cluster of $p$ processors, where $p=2^{i}$ for some integer $i$. Using the hierarchical partitioning strategy, the number of partitions for clusters at level $l$ is chosen as

$$
p_{l, c}=\max \left\{\frac{p}{2^{(l-1)}}, 1\right\}=\max \left\{p 2^{(1-l)}, 1\right\} .
$$

Then, the number of clusters assigned to each processor can be approximated as

$$
N_{l}^{p} \approx \frac{N_{l}}{p_{l, c}} \approx\left\{\begin{array}{rr}
2^{(1-l)} N_{1} / p, & l \leq \log _{2}(p) \\
4^{(1-l)} N_{1}, & l>\log _{2}(p)
\end{array}\right\} .
$$

In addition, samples of the fields are divided into

$$
p_{l, s}=\frac{p}{p_{l, c}}=\min \left\{2^{(l-1)}, p\right\}
$$

partitions along the $\theta$ direction for level $l$. For each cluster, the total number of samples per processor can be written as

$$
S_{l}^{p} \approx\left\{\begin{aligned}
2^{(l-1)} S_{1}, & l \leq \log _{2}(p) \\
4^{(l-1)} S_{1} / p, & l>\log _{2}(p)
\end{aligned}\right\} .
$$

By distributing both clusters and field samples, the hierarchical strategy improves the loadbalancing of the workload among processors. In addition, the communication time is significantly reduced compared to the previous parallelization strategies.

\section{Numerical Results}

The improved efficiency provided by the hierarchical strategy was demonstrated on relatively small problems involving millions of unknowns solved on 2 to 128 processors [2]. In this study, we use the hierarchical parallelization of MLFMA for the solution of very large electromagnetics problems. As an example, we present the solution of scattering problems involving various metallic objects depicted in Fig. 1. These are (a) a sphere of radius $180 \lambda$, (b) the NASA Almond of length $715 \lambda$, (b) a $400 \lambda$ long wing-shaped object with sharp edges and corners, and (d) the stealth airborne target Flamme with a maximum dimension of $720 \lambda$. All objects are discretized with $\lambda / 10$ triangles, leading to matrix equations with more than 100 million unknowns. Iterative solutions are performed by using the biconjugate-gradient-stabilized (BiCGStab) algorithm accelerated via MLFMA with two 


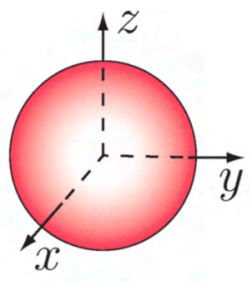

(a)

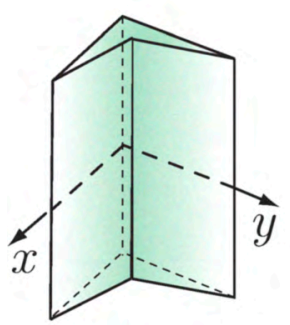

(c)

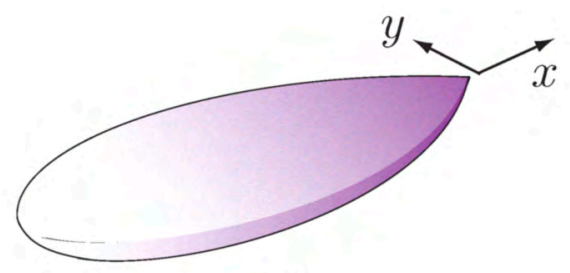

(b)

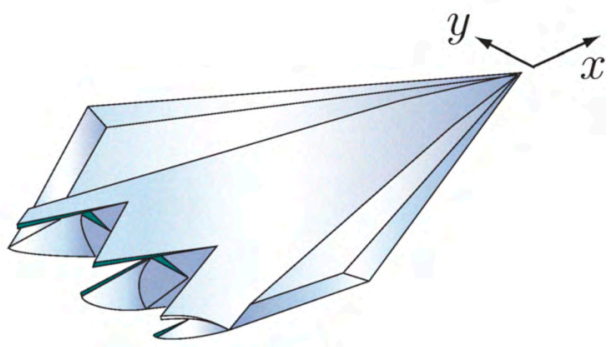

(d)

Fig. 1. Examples to metallic objects considered in this study: (a) Sphere, (b) NASA Almond, (c) wing-shaped object, and (d) Flamme.

Table 1: Solutions of Large-Scale Scattering Problems

\begin{tabular}{|c|c|c|c|c|c|c|c|}
\hline & & & & \multicolumn{2}{c|}{ 16 Processors } & \multicolumn{2}{c|}{ 64 Processors } \\
\hline Problem & Size & Unknowns & $\begin{array}{c}\text { BiCGStab } \\
\text { Iterations }\end{array}$ & $\begin{array}{c}\text { Time } \\
\text { (minutes) }\end{array}$ & $\begin{array}{c}\text { Memory } \\
\text { (GB) }\end{array}$ & $\begin{array}{c}\text { Time } \\
\text { (minutes) }\end{array}$ & $\begin{array}{c}\text { Memory } \\
\text { (GB) }\end{array}$ \\
\hline Sphere & $360 \lambda$ & $135,164,928$ & 23 & 975 & 424 & 292 & 467 \\
\hline Wing & $400 \lambda$ & $121,896,960$ & 17 & 546 & 431 & 162 & 500 \\
\hline Almond & $715 \lambda$ & $125,167,104$ & 20 & 769 & 385 & 215 & 471 \\
\hline Flamme & $720 \lambda$ & $134,741,760$ & 44 & 1186 & 427 & 345 & 513 \\
\hline
\end{tabular}

digits of accuracy. Convergence of the iterative solutions are also accelerated by using block-diagonal preconditioners. Solutions are performed on a parallel cluster, which consists of 16 computing nodes. Each node involves $3.0 \mathrm{GHz}$ Intel Xeon processors and a total of $32 \mathrm{~GB}$ memory. We parallelize the solutions into 16 and 64 processes by employing 1 and 4 processors per node. Table I lists the number of iterations (for $10^{-3}$ residual error), peak memory, and the total processing time including setup and solution parts. Using 64 processors, the parallelization efficiency for both processing time and memory is more than $80 \%$ with respect to 16 processors. Due to this relatively high efficiency provided by the hierarchical partitioning strategy, we are able to perform all four solutions in Table I in 1014 minutes, which is less than 17 hours.

Efficient solutions of the large problems presented in Table I are obtained under strict conditions in terms of accuracy. We note that larger problems could be solved by sacrificing the accuracy. Solutions can be relaxed by using coarser discretizations, reducing the truncation numbers, or decreasing the order of interpolations. The size of the problems can also be enlarged at the cost of increasing processing time. For example, we calculate and store radiation and receiving patterns of basis and testing functions during the setup of the program, and we use them efficiently during iterations. Calculating the patterns on the fly in each matrix-vector multiplication without storing them would increase the processing time, but 
larger problems could be solved. In general, we do not follow these tricks since the major purpose of this study is solving very large electromagnetics problems fast and accurately.

Finally, to demonstrate the accuracy of the solutions, Fig. 2 presents bistatic radar cross section (RCS) values for a sphere of radius $210 \lambda$. In Fig. (a), the normalized RCS (RCS $/ \pi a^{2}$, where $a$ is the radius of the sphere in meters) is plotted in decibels (dB), where $0^{\circ}$ and $180^{\circ}$ correspond to the back-scattering and forward-scattering directions, respectively. Computational values obtained by solving a 204,823,296-unknown matrix equation are compared with the analytical values obtained by a Mie-series solution. For an easy comparison, Fig. (b) presents the same results from $175^{\circ}$ and $180^{\circ}$. We observe that the computational and analytical results perfectly agree with each other.

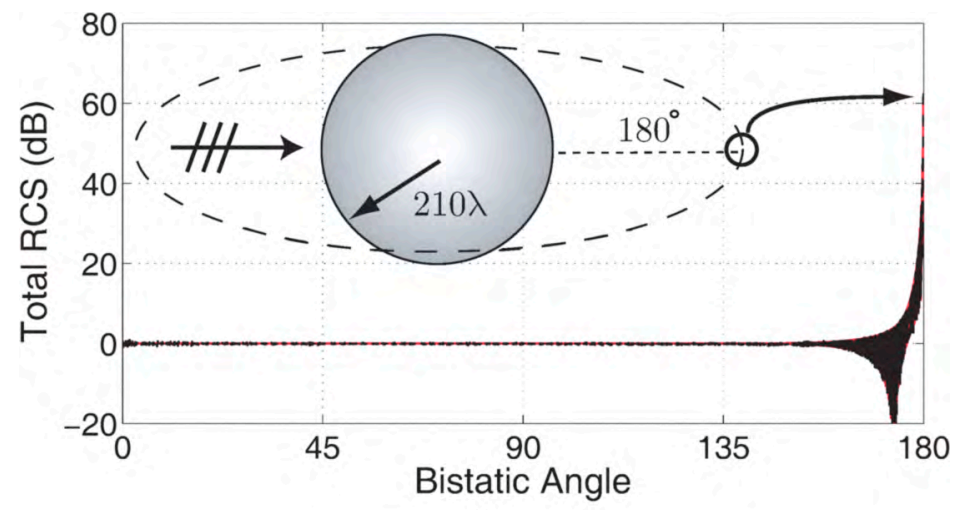

(a)

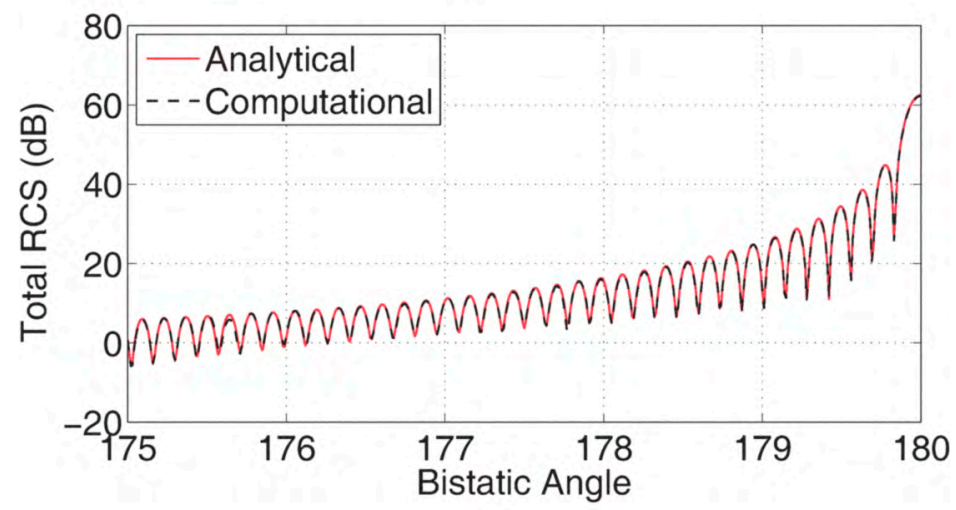

(b)

Fig. 2. Bistatic RCS (in $\mathrm{dB}$ ) of a sphere of radius $210 \lambda$ (a) from $0^{\circ}$ to $180^{\circ}$ and (b) from $175^{\circ}$ to $180^{\circ}$, where $180^{\circ}$ corresponds to the forward-scattering direction.

\section{References}

[1] J. Song, C.-C. Lu, and W. C. Chew, "Multilevel fast multipole algorithm for electromagnetic scattering by large complex objects," IEEE Trans. Antennas Propagat., vol. 45, no. 10, pp. 1488-1493, Oct. 1997.

[2] Ö. Ergül and L. Gürel, "Hierarchical parallelisation strategy for multilevel fast multipole algorithm in computational electromagnetics," Electron. Lett., vol. 44, no. 1, pp. 3-5, Jan. 2008. 